\title{
The 4F2 Heavy Chain Gene: a Molecular Model of Inducible Gene Expression in Human T Cells
}

\author{
Jeffrey M. Leiden, Li-Huan Yang, Gerald D. Morle, Car1 H. June, \\ Tullia Lindsten, Craig B. Thompson and Beverly Karpinski
}
The Howard Hughes Medical Institute and the Departments of Internal Medicine and Microbiology/Immunology, University of Michigan Medical Center, Ann Arbor, MI 48109, USA

\begin{abstract}
We have utilized the human 4F2 heavy chain (4F2HC) gene as a model system in studies designed to elucidate the molecular events involved in regulating inducible gene expression during normal human $T$-cell activation. In previous studies we have shown that steady state levels of 4F2HC mRNA are induced 50-60-fold within $6 \mathrm{~h}$ of $\mathrm{T}$-cell activation by phytohemagluttinin (PHA) and that the induction of 4F2HC gene expression involves both the protein kinase $C$ and calcium-mediated activation pathways. Despite the fact that the $4 F 2 H C$ gene is highly regulated in $T$ cells, the $5^{\prime}$ upstream region of the 4F2HC gene contains a housekeeping promoter which is $G+C$ rich, lacks TATA or CCAAT sequences, and contains four potential binding sites for the ubiquitous Sp1 transcription factor. The major regulatory elements of the 4F2HC gene do not reside within this $5^{\prime}$ upstream region but instead, map to the exon 1-intron 1 region of the gene. The low levels of mature 4F2HC mRNA in resting $T$ cells result from a block to transcription elongation within the exon 1-intron 1 region of the gene rather than promoter inactivity. Phorbol ester stimulation of resting $T$ cells induces 4F2HC gene expression by removing this block to transcription elongation. We now report that in addition to its ability to serve as a transcriptional attenuator, the 4F2HC first intron contains a powerful enhancer element which is active in a wide variety of cell types including malignant human $T$ cells. Full enhancer activity is displayed by a 186 bp fragment of the first intron which contains binding sites for two novel nuclear proteins (NF-4FA and NF-4FB) which flank a consensus binding site for the AP-1 transcription factor. A cDNA encoding the NF-4FB enhancer binding protein has been cloned by screening a lambda gt11 cDNA library with a rabiolabelled oligonucleotide corresponding to the NF-4FB recognition sequence.
\end{abstract}

Correspondence should be addressed to Dr J. Leiden, HHMI, MSRBI Rm 4510. 1150 West Medical Campus Drive, University of Michigan Medical Center, Ann Arbor, MI 48109, USA. 


\section{Introduction}

Antigen recognition by the heterodimeric $\alpha / \beta$ receptor of human $T$ lymphocytes results in a complex and precisely orchestrated set of changes in T-cell gene expression including the induction of a set of nuclear proto-oncogenes $[1,2]$, the production of a variety of soluble lymphokines [3], and the expression of a novel group of cellsurface antigens [4]. While a great deal has recently been learned about the structural requirements of antigen recognition by the TCR $\alpha / \beta$ molecule (reviewed in [5]), relatively less is understood about the molecular mechanisms involved in modulating coordinate gene expression during T-cell activation. Cross-linking of the TCR $\alpha / \beta$ CD3 complex has been shown to result in phosphoinositol turnover, protein kinase $\mathrm{C}$ activation, and transient increases in cytosolic ionized calcium levels (reviewed in [6]). How these (and other) second messengers are subsequently able to modulate $T$-cell gene expression remains unclear.

We have utilized the $4 \mathrm{~F} 2$ cell-surface antigen as a model system of gene regulation during human $\mathrm{T}$-cell activation. $4 \mathrm{~F} 2$ is a $120 \mathrm{kD}$ disulphide-linked heterodimer composed of an $85 \mathrm{kD}$ glycosylated heavy chain (4F2HC) and a $35 \mathrm{kD}$ non-glycosylated light chain (4F2LC) [7]. 4F2 is of interest in studies of T-cell activation because it is a T-cell activation antigen which is expressed at low levels on resting peripheral blood $\mathrm{T}$ cells, but rapidly induced following activation with either lectins or antigen [4]. Increases in $4 \mathrm{~F} 2$ cell-surface expression first occur $4 \mathrm{~h}$ after lectin stimulation, and peak at 16-20 h, well before the onset of DNA synthesis or the appearance of the interleukin-2 (IL-2) receptor $\alpha$ chain [4]. In addition, 4F2 appears to be intimately involved in the process of $\mathrm{T}$-cell activation because monoclonal antibodies directed against the $4 \mathrm{~F} 2 \mathrm{HC}$ are able to block efficiently the proliferation of cultures of activated T cell in vitro [8].

We have previously reported that the $4 \mathrm{~F} 2 \mathrm{HC}$ is encoded by an 1862 bp cDNA [9]. DNA sequence analysis of the $4 \mathrm{~F} 2 \mathrm{HC}$ cDNA demonstrated that it encodes a 529 amino acid type II membrane glycoprotein composed of an 81 aa $\mathrm{N}$-terminal intracytoplasmic domain, a single hydrophobic putative transmembrane region, and a 425 aa extracellular domain. The $4 \mathrm{~F} 2 \mathrm{HC}$ is encoded by a single copy human (and murine) gene and does not display significant homology with other known proteins [9]. In order to investigate the molecular mechanisms which regulate $4 \mathrm{~F} 2 \mathrm{HC}$ gene expression in resting, activated, and malignant $T$ cells we have recently reported the isolation and characterization of a $4 \mathrm{~F} 2 \mathrm{HC}$ genomic clone [10]. The $4 \mathrm{~F} 2 \mathrm{HC}$ gene is composed of nine exons and spans $8 \mathrm{~kb}$ of the long arm of human chromosome 11 [10]. Despite our findings that the gene is highly regulated during $\mathrm{T}$-cell activation, the $5^{\prime}$ end of the gene contains a housekeeping promoter which is $\mathrm{G}+\mathrm{C}$ rich, lacks TATA or CCAAT sequence motifs and contains binding sites for the ubiquitous $\mathrm{Sp} 1$ transcription factor [11]. There are DNAaseI hypersensitive sites in both the $5^{\prime}$ upstream and first intron regions of the gene [10]. Taken together, these findings suggested that $4 \mathrm{~F} 2 \mathrm{HC}$ gene expression might be regulated by sequences internal to the gene.

Our previous studies of $4 \mathrm{~F} 2 \mathrm{HC}$ gene transcription in resting and activated $\mathrm{T}$ cells have demonstrated that the gene can be synergistically induced by phorbol esters which activate protein kinase $\mathrm{C}$ and the calcium ionophore, ionomycin [12]. The 4F2HC promoter was shown to be equally active in resting and phorbol ester activated human peripheral blood $T$ cells [12]. The low steady state levels of mature 
4F2HC mRNA found in resting $T$ cells was shown to be the result of a block to transcription elongation within the exon 1 -intron 1 region of the gene. Phorbol ester activation removes this block to transcription elongation resulting in increased levels of full-length 4F2HC mRNA [12]. This phorbol ester induced 4F2HC gene induction is inhibited by cycloheximide and therefore requires protein synthesis [12]. In a parallel set of studies, ionomycin treatment of resting $T$ cells was shown to increase 4F2HC promoter activity [12].

In order to map the sequences responsible for transcriptional attenuation in the $4 \mathrm{~F} 2 \mathrm{HC}$ gene we have introduced $4 \mathrm{~F} 2 \mathrm{HC}^{\prime}$-upstream, exon 1 , and intron 1 sequences into a variery of plasmids containing the bacterial chloramphenicol acetyltransferase (CAT) reporter gene. Surprisingly, we found that the $4 \mathrm{~F} 2 \mathrm{HC}$ first intron contains a potent enhancer element which is active in a wide variety of human and murine tissue culture cell lines. This enhancer element, which acts on a variety of promoters including the SV40 promoter/enhancer, the HSV thymidine kinase (TK) promoter, and the 4F2HC promoter has been mapped to a $186 \mathrm{bp} \mathrm{AluI/RsaI} \mathrm{fragment} \mathrm{within} \mathrm{the}$ 4F2HC first intron. The mapping studies have suggested that there are multiple enhancer motifs within this DNA fragment. DNAase footprinting and gel mobility shift studies revealed that this fragment contains binding sites for two different Jurkat nuclear proteins (NF-4FA and NF-4FB). These binding sites are distinct from previously described enhancer binding sires. In addition this enhancer fragment contains a consensus binding site for the AP-1 transcription factor. Using a modification of the technique of Singh et al. [13] we have cloned a cDNA which encodes the NF-4FB enhancer binding protein.

\section{Results}

\section{The $4 F 2 H C$ first intron contains a potent enhancer element}

In an attempt to map the sequences responsible for the previously reported [12] transcriptional attenuator activity of the $4 \mathrm{~F} 2 \mathrm{HC}$ gene exon 1 -intron 1 region a variety of genomic fragments were subcloned into the HindIII site of the pSV2cat [14] plasmid between the SV40 promoter/enhancer and the bacterial chloramphenicol acetyltransferase (CAT) reporter gene (Figure 1). These constructs were transfected into human Jurkat T cells using DEAE-dextran [10]. Somewhat surprisingly, the pSV2-I $\mathrm{I}_{1}$ cat plasmid containing a 509 bp $4 \mathrm{~F} 2 \mathrm{HC}$ genomic fragment which includes the entire 4F2HC first intron surrounded by short regions of exon 1 and exon 2 sequences in order to allow for accurate splicing (Figure 1; pSV2-I cat) displayed 60-fold higher CAT activity, compared with the pSV2cat plasmid alone (Figure 2). In order to determine whether this effect was due to an enhancer element, or, alternatively, to a cryptic promoter within this region of the 4F2HC gene, this same fragment was subcloned in both orientations into the BamHI site which lies downstream of the bacterial CAT gene in pSV2cat (Figure 1; pSV2I,Bam/cat and $\mathrm{pSV} 2 \mathrm{RI}_{1} \mathrm{Bam} / \mathrm{cat}$ ). Both constructs containing the $4 \mathrm{~F} 2 \mathrm{HC}$ first intron expressed 4045-fold more CAT activity than the pSV2cat control plasmid (Figure 2) indicating that this fragment contains a potent enhancer element. In order to determine the cellular specificity of this enhancer, the identical CAT constructs were used to transfect a variety of human and murine cell lines including K562 human erthroleukemia 


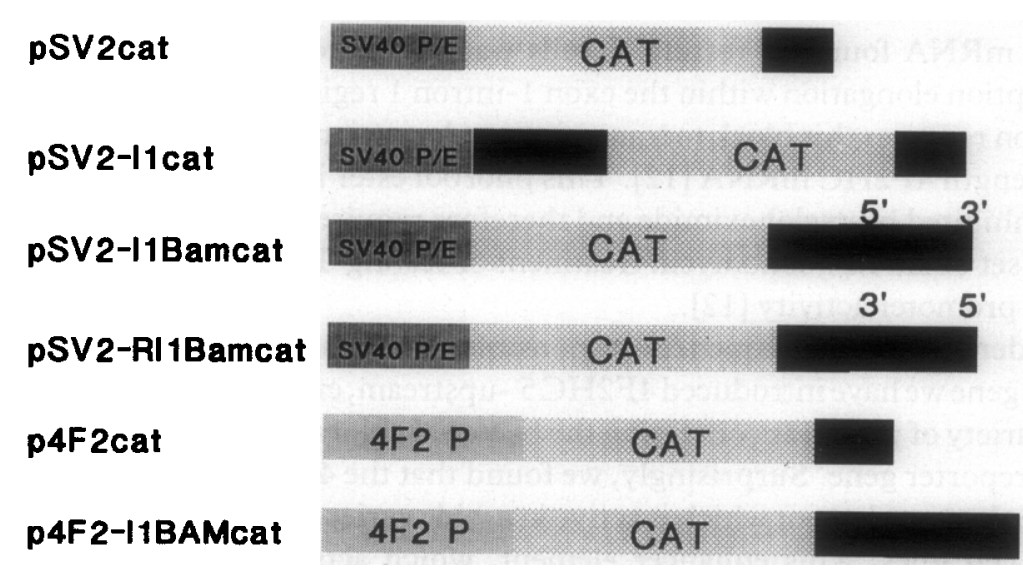

Figure 1. A schematic representation of CAT reporter plasmids. The $509 \mathrm{bp} \mathrm{StuI/SacI} \mathrm{fragment}$ containing the human $4 \mathrm{~F} 2 \mathrm{HC}$ first intron $\left(4 \mathrm{~F}_{2} \mathrm{I}_{1}\right)$ [10] was cloned into the previously described pSV2cat plasmid [14] either at the HindIII site located between the SV40 promoter/enhancer (SV40 P/E) and the bacterial CAT gene (pSV2-I, cat) or at the BamHI site located downstream of the bacterial CAT reporter gene in pSV2cat. Downstream constructs containing the 4 F2HC first intron in either a $5^{\prime}$ to $3^{\prime}$ (pSV2$I_{1}$ Bam cat) or a $3^{\prime}$ to $5^{\prime}$ (pSV2-RI, Bam cat) were obtained. Similar constructs (p4F2cat and p4F2-I Bam cat) were made by replacing the SV40 promoter/enhancer in the pSV2cat plasmid with a promoter fragment (4F2 P) (bp -589-+101) from the human 4F2HC 5' upstream region [10].

cells, JY human B cells, murine L cell fibroblasts, and murine EL4 T cells. In all cases the $4 \mathrm{~F} 2 \mathrm{HC}$ first intron was able to increase transcription from the SV40 promoter/enhancer by $2-55$-fold (Figure 2). However, it is worth noting that the enhancer was less active in both human B cells and mouse $L$ cells. Part of this difference was due to the fact that the SV40 promoter/enhancer displayed higher basal levels of CAT activity in these cells. In a parallel series of experiments, the $4 \mathrm{~F} 2 \mathrm{HC}$ first intron was also shown to function as a potent enhancer of both the 4F2HC and Herpes simplex virus (HSV) thymidine kinase (TK) promoters resulting in 5-10-fold increases in the activity of these promoters in Jurkat cells (data not shown). Taken together these experiments indicated that the first intron of the 4F2HC gene contains a powerful classical enhancer element which can function with a number of heterologous promoters in an orientation and position independent fashion in a wide variety of cell types. The potency of this enhancer is rather remarkable in that it is able to significantly increase transcription in $\mathrm{T}$ cells even in the presence of the SV40 enhancer element (Figure 2).

\section{Mapping of the $4 F 2 H C$ enhancer}

In order to map more precisely the enhancer element, a variety of restriction enzyme fragments from the $4 \mathrm{~F} 2 \mathrm{HC}$ first intron were subcloned into the BamHI site downstream of the bacterial CAT gene in pSV2cat and assayed for their ability to enhance CAT transcription in Jurkat cells (Figure 3). Fragments from the left half of the intron (bp 0-250) or the right half of the intron (bp 253-509) produced by digestion with NarI or HaeII displayed a marked reduction in enhancer function although both fragments retained the ability to enhance CAT expression by $10-15$ fold as compared to the pSV2cat control plasmid. In contrast the $186 \mathrm{bp} \mathrm{AluI/RsaI} \mathrm{frag-}$ ment from the middle of the intron displayed full enhancer activity (Figure 3 ). These 

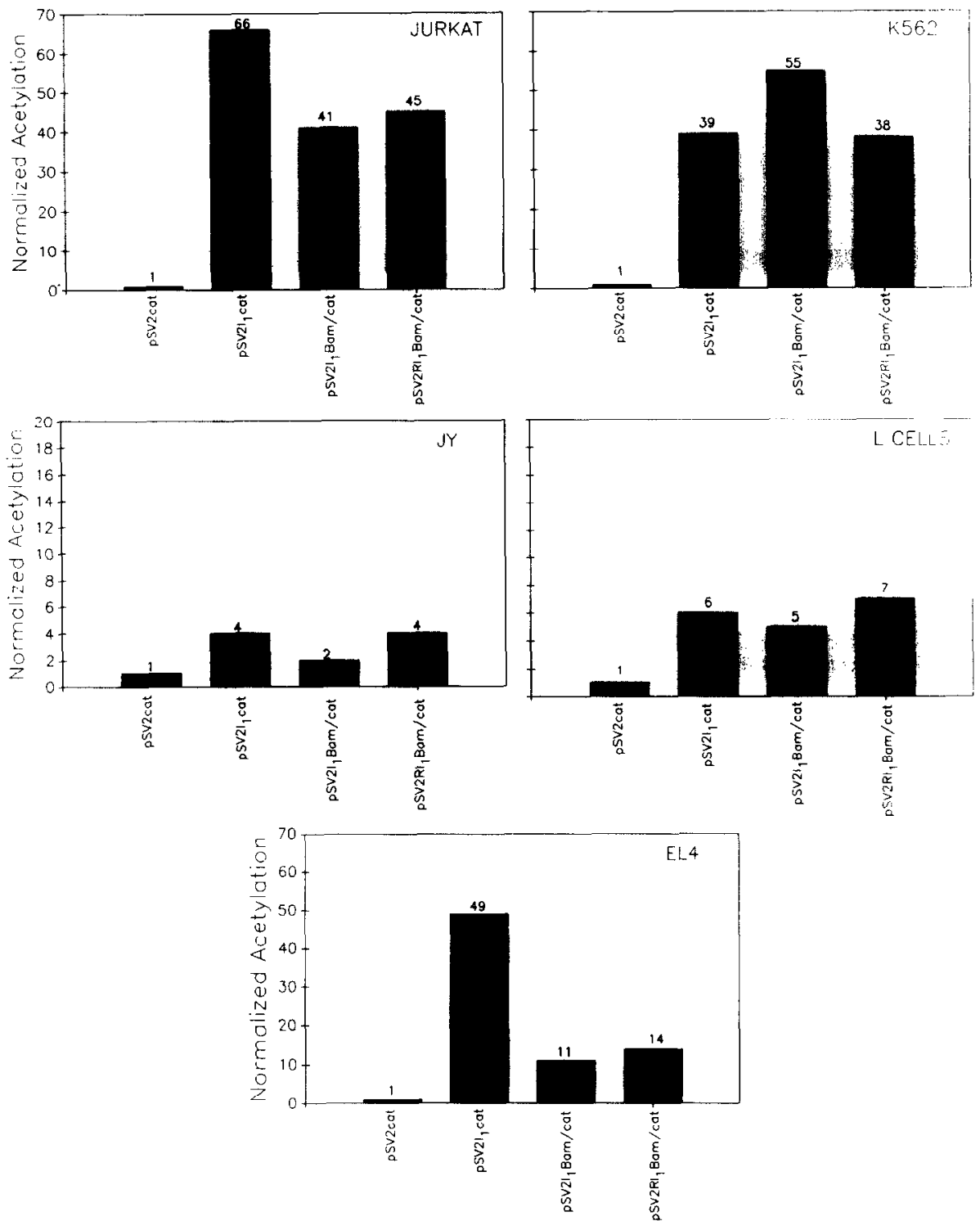

Figure 2. The effects of the 4F2 heavy chain first intron on SV40 promoter/enhancer activity in a variety of human and murine cell lines. The pSV2cat, pSV2-I cat, pSV2-I,Bam/cat, and pSV2-RI Bam/cat plasmids (see Figure 1) were used to transfect human Jurkat T cells, K562 erythroleukemia cells, and JY B cells as well as murine L-cell fibroblasts and EL4 T cells using a modification of the DEAE-dextran approach as previously described [10]. Forty-eight hours following transfection, cells were harvested and cell extracts normalized for protein content were assayed for CAT activity using standard techniques and thin layer chromatography [14]. Spots containing acetylated chloramphenicol were cut from the TLC plates and quantitated by liquid scintillation counting. The CAT activity produced by a control pSV2 cat plasmid was normalized to a value of 1 in each of the experiments. 

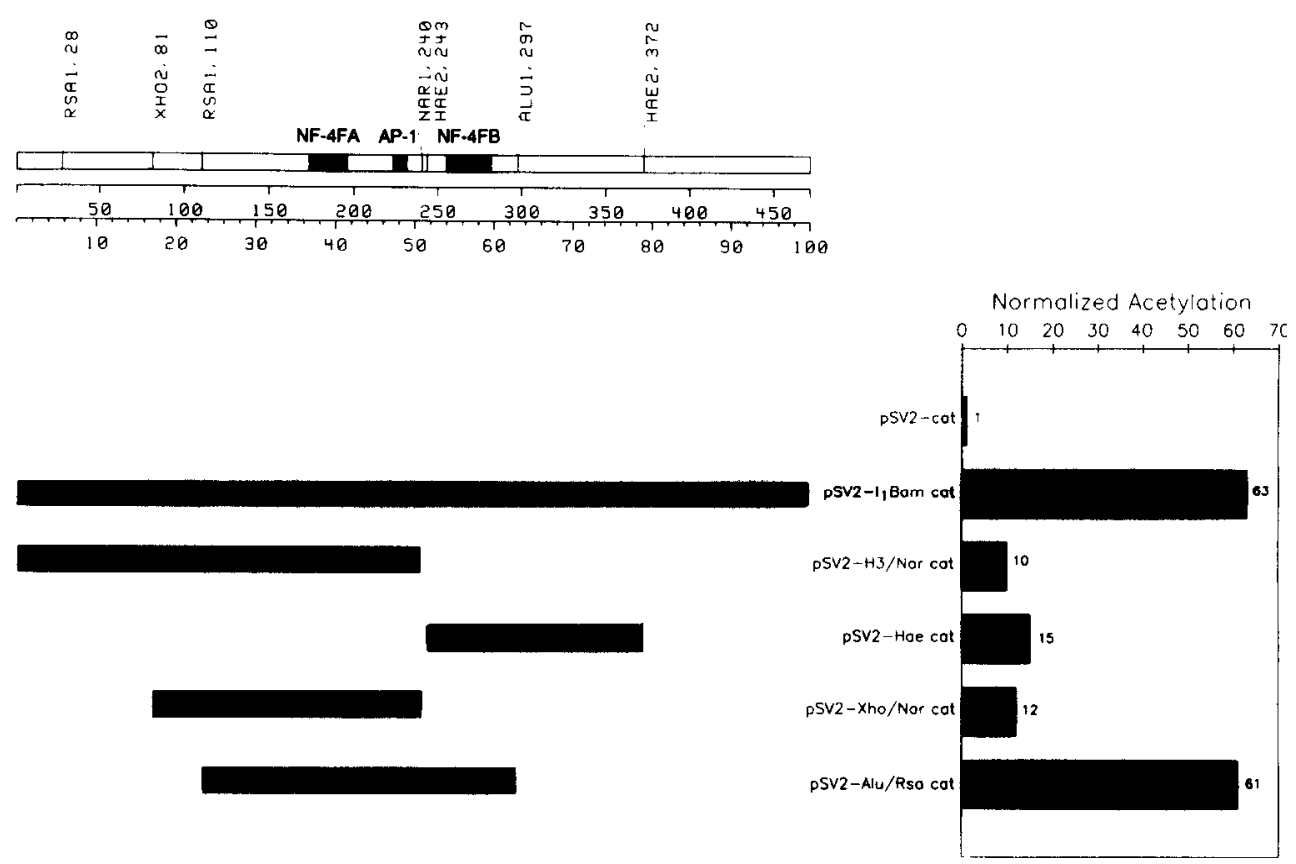

Figure 3. Mapping of the 4F2HC first intron enhancer element. Restriction enzyme fragments derived from the human 4F2HC first intron were cloned into the BamHI site downstream of the bacterial CAT gene in the pSV2cat plasmid vector. These constructs were used to transfect human Jurkat cells as described in the legend to Figure 2. Two days following transfection, the cell extracts were assayed for bacterial CAT activity as described in the legend to Figure 2. As an internal control, the CAT activity produced by the pSV2cat plasmid was normalized to a value of 1 . The upper left panel shows a partial restriction endonuclease map of the human 4F2HC first intron. Restriction enzyme fragments used in the CAT constructs are shown by rectangular black bars in the lower left panel. The CAT activity produced by each of these constructs is shown as normalized acetylation in the bottom right hand graph. The NF4FA, AP-1 and NF-4FB nuclear protein binding sites as described in the legend to Figure 4 are shown in the restriction endonuclease map in the upper left panel.

results demonstrated that while all of the enhancer sequences map to a relatively small fragment from the center of the intron, sequences on both sides of the NarI site (bp 250) are needed for full enhancer activity. Thus either the NarI site lies in the middle of a single enhancer motif or there are multiple enhancer motifs which flank this site.

\section{The $4 F 2 H C$ enhancer contains two novel nuclear protein binding sites}

In order to identify putative binding sites for $4 \mathrm{~F} 2 \mathrm{HC}$ enhancer binding proteins, restriction enzyme fragments from the 4F2HC first intron were subjected to DNAaseI footprint analyses using nuclear extracts prepared from Jurkat $T$ cells (Figure 4). Two sites (NF-4FA and NF-4FB) within the AluI/RsaI enhancer fragment were specifically protected by Jurkat nuclear proteins. Interestingly these two sites surround a consensus binding site for the AP-1 transcription factor $[15,16]$ which is not footprinted by the unstimulated Jurkat nuclear extracts. It is also worth noting that these two sites lie on either side of the NarI site which was shown to bisect 

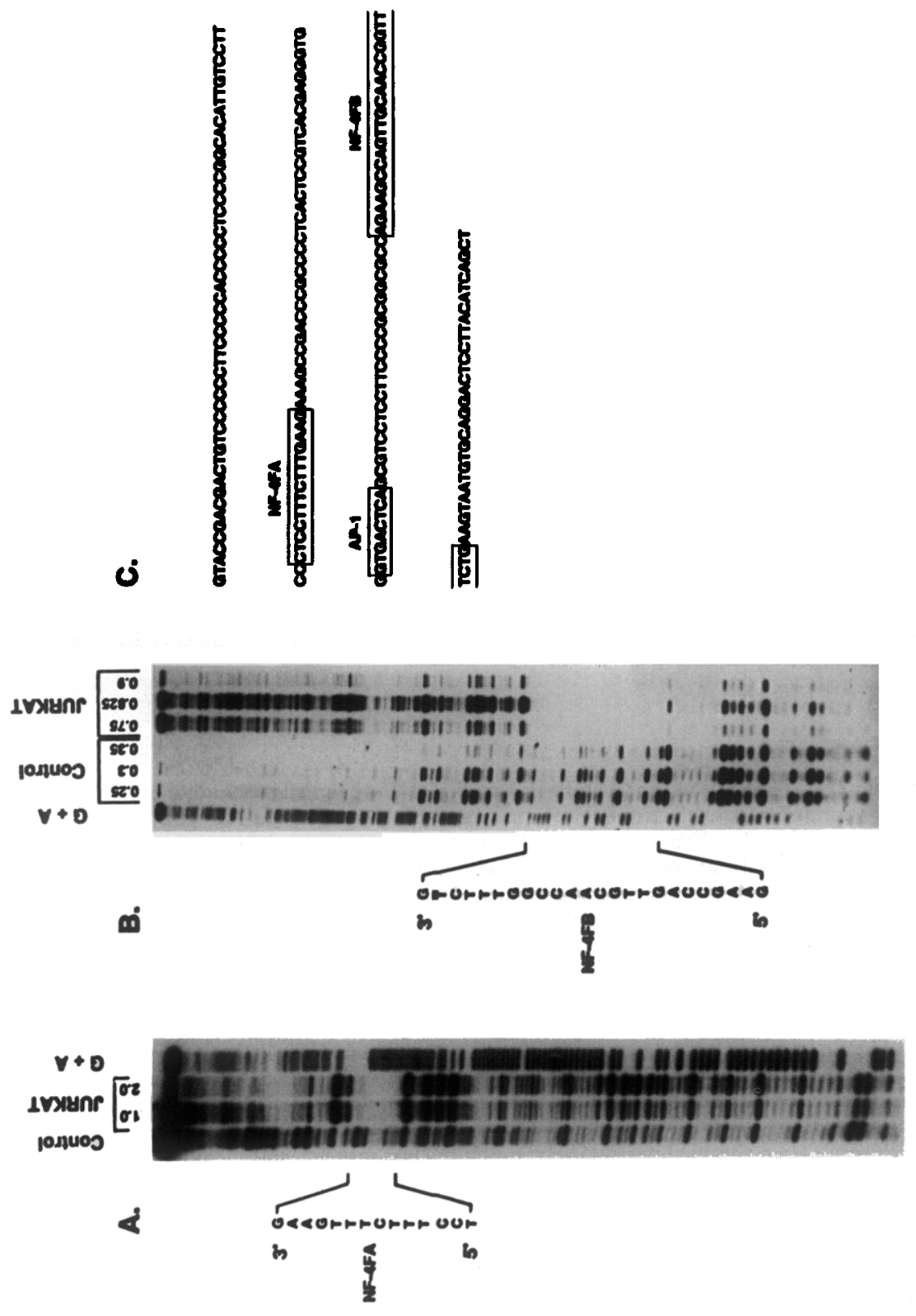

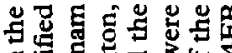
它

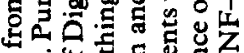

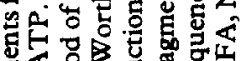
通焉焉出

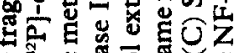

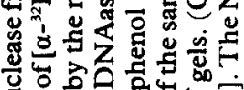

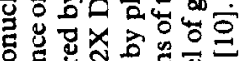

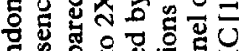

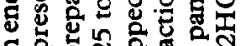

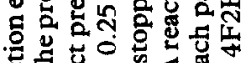

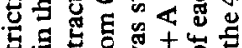

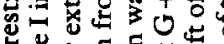

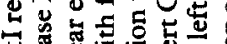

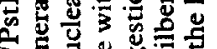
政总宁

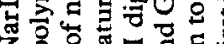

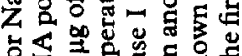

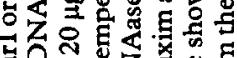

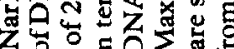

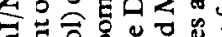

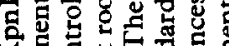
E

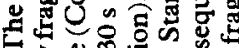

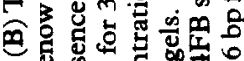

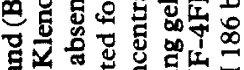

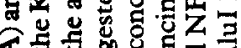

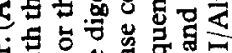

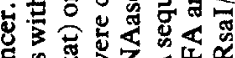

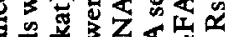

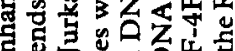

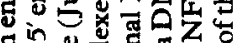

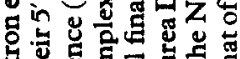

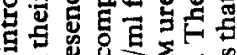

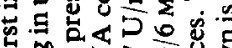

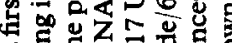

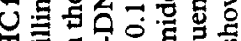

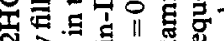

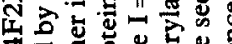

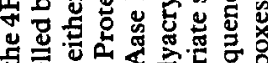

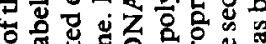

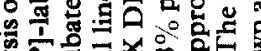

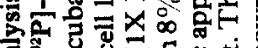

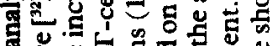

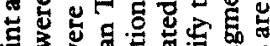

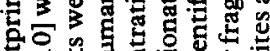

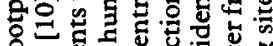

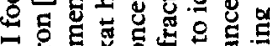

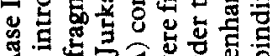

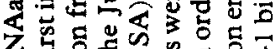

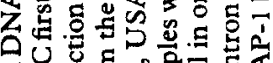

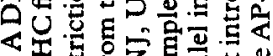

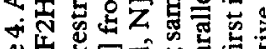

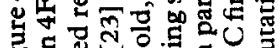

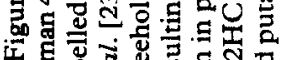

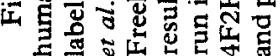


A.

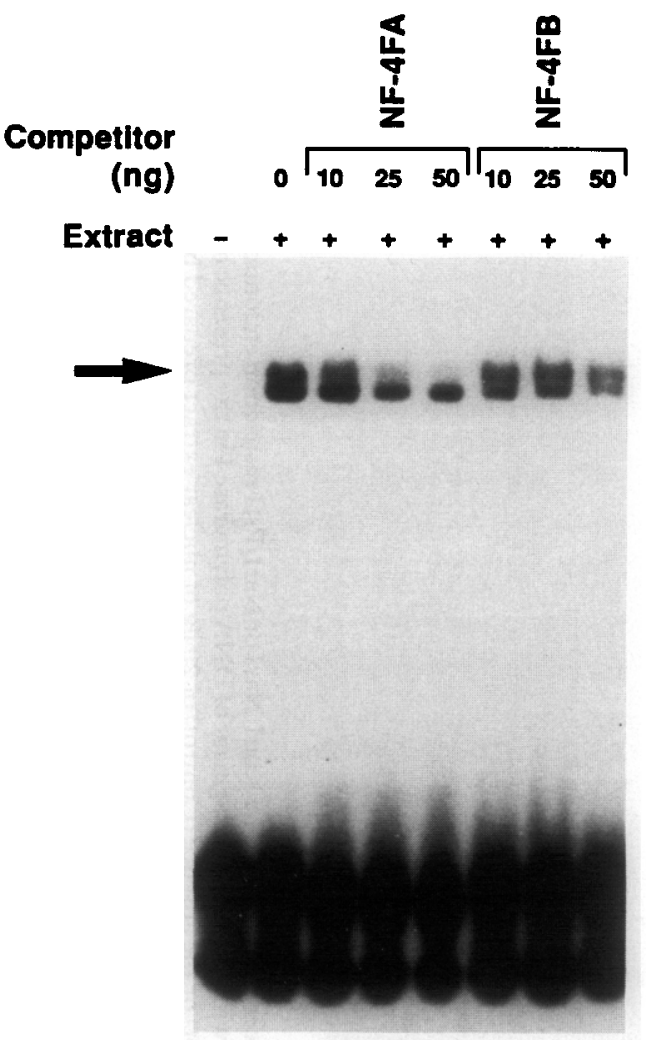

NF-4FA
B.

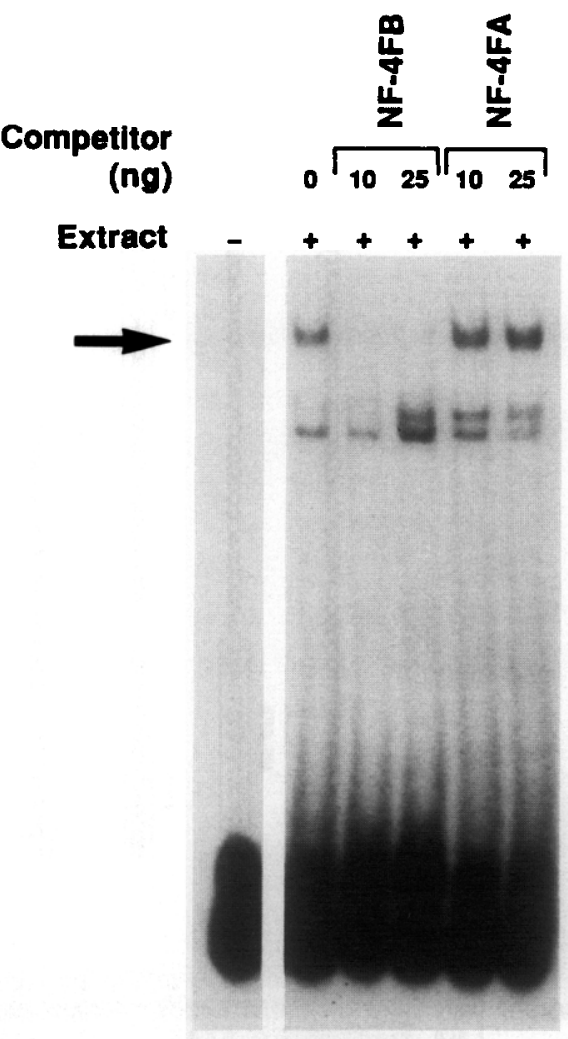

NFAFB

Figure 5. A gel mobility shift analysis of the (A) NF-4FA and (B) NF-4FB nuclear binding proteins. Complimentary single-stranded oligonucleotides corresponding to the NF-4FA and NF-4FB nuclear protein binding sites as determined in Figure 4 were synthesized using an Applied Biosystems Model 380B DNA synthesizer. For the sake of convenience, overhanging BamHI and Bg1II single-stranded compatible ends were included in the appropriate oligonucleotides. Double-stranded oligonucleotides were formed by annealing under the appropriate salt and temperature conditions and were labelled using the Klenow fragment of DNA polymerase I in the presence of $\left[\alpha-{ }^{32} \mathrm{P}\right]-\mathrm{dATP}$. Labelled oligonucleotides were purified by electrophoresis in $10 \%$ polyacrylamide gels. Binding reactions were as previously described [24] and contained $2 \mu \mathrm{g}$ of Jurkat nuclear protein extract, $10,000 \mathrm{cpm}$ of labelled oligonucleotide, $0.5 \mu \mathrm{g}$ of polydI:dC (Pharmacia, Piscataway, NJ, USA) in a total volume of $15 \mu \mathrm{l}$. Complexes were allowed to form for $15 \mathrm{~min}$ at room temperature and were then fractionated on $4 \%$ non-denaturing polyacrylamide gels as previously described [24]. In cold competitor experiments, complexes were allowed to form in the presence of 10-50 ng (20-100-fold molar excess) of unlabelled double-stranded cold NF-4FA or NF-4FB competitor. Arrows to the left of each set of gels indicate the positions of bands of altered mobility which result from specific interactions of Jurkat nuclear proteins and the NF-4FA and NF-4FB double-stranded oligonucleotide probes.

the enhancer elements present in this fragment (see Figure 3). In order to confirm the specificity of the NF-4FA and NF-4FB proteins, synthetic oligonucleotides corresponding to the two binding sites were synthesized and used in gel mobility shift assays with Jurkat nuclear extracts (Figure 5). Jurkat nuclear extracts contained several proteins which were able to bind to the NF-4FA and NF-4FB oligonucleotides and cause a shift in their electrophoretic mobility. Cold competitor experiments 
demonstrated that the formation of one of the shifted NF-4FA complexes [shown by the arrow in Figure 5(A)] was inhibited by a 50-100-fold molar excess of specific unlabelled NF-4FA cold competitor oligonucleotide, but not by an identical amount of cold NF-4FB competitor. Similarly, the formation of one of the shifted NF-4FB complexes [shown by the arrow in Figure 5(B)] was inhibited by excess cold NF4 FB competitor but not by an identical amount of cold NF-4FA competitor. These results indicated that the NF-4FA and NF-4FB sequences specifically bind distinct Jurkat nuclear proteins. The functional importance of the NF-4FA, NF-4FB, and AP-1 sites was underscored by the finding that all three sites are highly conserved in the murine $4 \mathrm{~F} 2 \mathrm{HC}$ first intron which has similar enhancer activity (data not shown), while the remainder of the murine and human introns display a significantly lower level of homology (data not shown).

\section{Molecular cloning of a $c D N A$ encoding the NF-4FB enhancer binding protein}

In order to understand better the function of the $4 \mathrm{~F} 2 \mathrm{HC}$ first intron enhancer, we have attempted to clone a cDNA encoding the NF-4FB nuclear binding protein. A random hexanucleotide-primed lambda gt11 cDNA library from the K562 human erythroleukemia cell line was screened with a multimeric ${ }^{32} \mathrm{P}$-labelled oligonucleotide probe corresponding to the NF-4FB binding site using a modification of the technique described by Singh et al. [13] and Staudt et al. [17] (Figure 6). Multimeric probe was constructed by labelling single-stranded oligonucleotides with $\left[\gamma-{ }^{32} \mathrm{P}\right]$ ATP using T4 polynucleotide kinase, annealing the labelled single-stranded oligonucleotides, and then ligating the annealed oligonucleotides prior to use in the screening protocol. This technique was found to produce probes of significantly higher specific activity than the labelling technique originally described by Singh $e t$ al. using the Klenow fragment of DNA polymerase I [13]. Four of the 750,000 recombinant clones screened produced fusion proteins which bound to the NF-4FB oligonucleotide. Of these, three displayed specific binding as they were unable to bind similarly prepared oligonucleotide probes corresponding to the NF-4FA binding site (Figure 7). One of the clones encoded a non-specific DNA binding protein which bound equally to the NF-4FA and NF-4FB probes.

\section{Discussion}

We have utilized the $4 \mathrm{~F} 2 \mathrm{HC}$ gene as a model system of coordinate gene regulation following $T$-cell activation. Studies of the structure of the gene as well as its patterns of transcription have demonstrated that the gene contains a constitutively active housekeeping promoter which includes a calcium responsive element as well as four binding sites for the ubiquitous $\mathrm{Sp} 1$ transcription factor $[10,12]$. The major regulatory domains of the gene appear to map to the exon 1 -intron 1 region. $4 \mathrm{~F} 2 \mathrm{HC}$ gene expression in resting $T$ cells is down-regulated by a block to transcription elongation located between exon 1 and exon 2 . Induction of 4 F2HC gene expression by phorbol esters results from the removal of this block to transcription elongation [12].

The first intron of the $4 \mathrm{~F} 2 \mathrm{HC}$ gene also contains a powerful enhancer element which is active upon a number of heterologous promoters in a wide variety of cell types. Full enhancer activity is displayed by a $186 \mathrm{bp}$ fragment which contains 
1. Kinase Single Stranded Oligos $-32 P$

$32 \mathrm{P}$

\section{Anneal and Ligate Oligos}

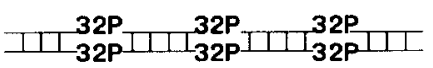

\section{Screen Lambda gt11 cDNA Expression Library}
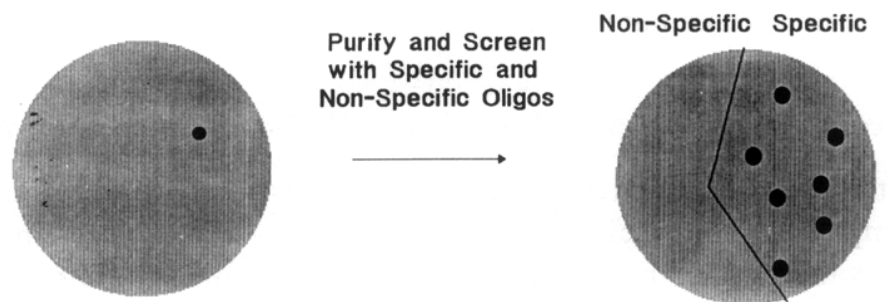

Figure 6. A schematic illustration of the cloning of a CDNA encoding the NF-4FB nuclear binding protein. Complimentary single-stranded oligonucleotides corresponding to the NF-4FB protein binding site were labelled in vitro with $\left[\gamma{ }^{32} \mathrm{P}\right]-\mathrm{ATP}$ using T4 polynucleotide kinase. The complimentary oligonucleotides were annealed in solution and then ligated using their BamHI and BglII compatible ends for $2 \mathrm{~h}$ at room temperature with 10 units of T4 DNA ligase. The ligated multimer probe was purified by passage over an elutip-d column (Schleicher and Schuell, Keene, NH). A random hexanucleotide primed $\lambda$ gt1 1 cDNA library prepared from the $\mathrm{K} 562$ human erythroleukemia cell line was the generous gift of Dr Francis Collins. Fifteen $82 \times 100 \mathrm{~mm}$ filters each containing 50,000 recombinant clones were screened in a total volume of $45 \mathrm{ml}$ with the NF-4FB labelled oligonucleotide probe using the method of Staudt $e t$ al. [17]. Positive clones were purified to homogeneity by repeated screening and then rescreened using either the non-specific (NF-4FA) or the specific (NF-4FB) labelled oligomeric probes.

tains two nuclear protein binding sites flanking a consensus AP-1 binding site. The importance of each of these sites in determining enhancer activity remains unclear. However, it is likely that at least two of the binding sites, (NF-4FB and AP-1 and/or NF-4FA) are required for full enhancer activity because cleavage of the enhancer fragment with NarI which separates the NF-4FB binding site from the AP-1 and NF-4FA sites results in a significant decrease in enhancer activity. The importance of the AP-1 site in determining enhancer activity is uncertain given our inability to footprint this site with nuclear extracts from Jurkat cells which display prominent enhancer activity. On the other hand, it is possible that low levels of AP-1 activity (which are not detectable in crude Jurkat nuclear extracts) are sufficient to activate the enhancer via this site. It is interesting to note that the AP-1/c-jun transcriptional activator has recently been shown to be induced at the mRNA level during serummediated activation of NIH3T3 cells [18], raising the possibility that AP-1/c-jun induction during $\mathrm{T}$-cell activation may be important in activating the 4F2HC enhancer element in normal human $T$ cells. Preliminary experiments have indicated that the NF-4FA protein is not expressed in K562 cells which display high levels of enhancer activity (see Figure 3) raising the possibility that this factor does not play an essential role in enhancer function. A final determination of the importance of each of 


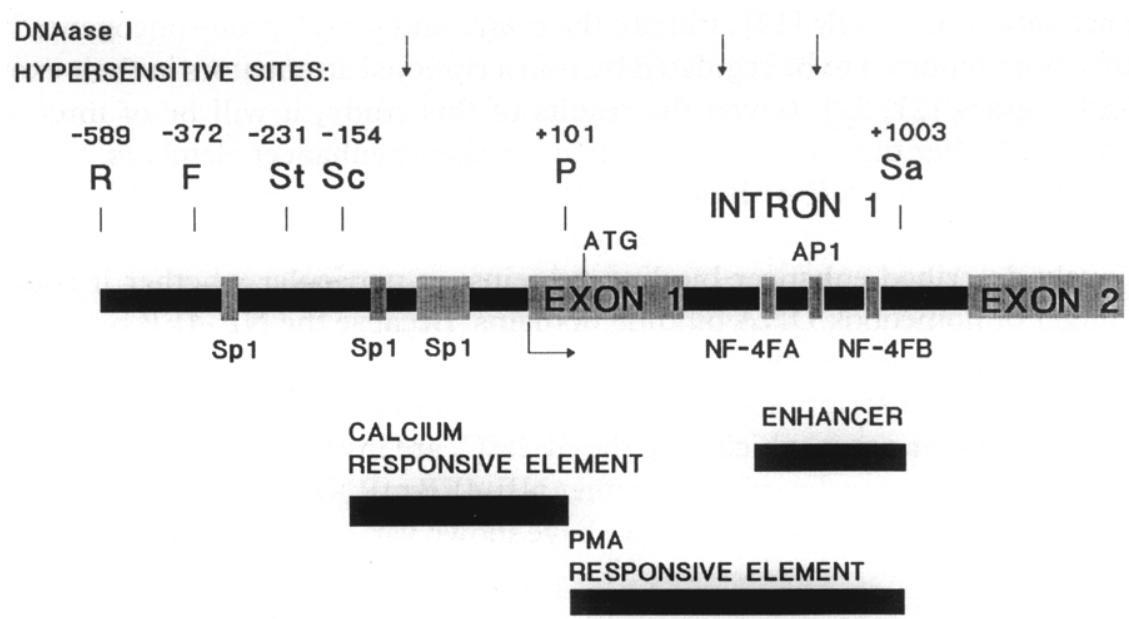

Figure 7. A schematic illustration of the regulatory elements of the human $4 F 2 H C$ gene. DNAase I hypersensitive sites within the $4 F 2 \mathrm{HC}$ gene in resting and activated peripheral blood $\mathrm{T}$ cells are shown in the top line of the Figure [10]. EcoRI (R), FokI (F), Stu (St), Sca (Sc), PstI (P), and SacI (Sa) restriction endonuclease sites as well as their relative map positions are shown in the second line of the figure [10]. The exon/intron structure of the gene as well as potential binding sites for the Sp1, AP1, NF-4FA, and NF$4 F B$ nuclear binding proteins are shown in the third line of the figure. The horizontal arrow indicates the transcription initiation site [10]. The location of the previously reported calcium responsive element and PMA responsive element (transcriptional attenuator) [12] as well as the newly described enhancer element are shown as horizontal bars on the bottom of the Figure.

these sequence motifs awaits ongoing experiments designed to test the effects of deletion and mutation of each of the individual binding sites on enhancer function. Finally, it should be noted that the $4 \mathrm{~F} 2 \mathrm{HC}$ enhancer is able to increase significantly levels of gene expression from both the SV40 promoter/enhancer and the HSVTK promoter, making it a useful adjunct in a variety of eukaryotic expression vectors (B. Oakley and J. Leiden, unpublished results).

The relationship between the enhancer element and the previously described transcriptional attenuator in the $4 \mathrm{~F} 2 \mathrm{HC}$ first intron remains unclear. Preliminary experiments indicate that the sequences responsible for transcriptional attenuation of $4 \mathrm{~F} 2 \mathrm{HC}$ gene transcription may map to the $5^{\prime}$ end of the first exon (data not shown). It is possible that inactivity of the $4 \mathrm{~F} 2 \mathrm{HC}$ enhancer in resting $\mathrm{T}$ cells results in an increased probability of premature transcription termination possibly due to an exon 1 sequence element which is difficult for the RNA polymerase complex to traverse. $T$-cell activation (via the protein kinase $C$ pathway) might result in enhancer activation which, in turn, might stabilize the RNA polymerase complex resulting in transcriptional readthrough and increased levels of mature $4 \mathrm{~F} 2 \mathrm{HC}$ mRNA. In testing this model, it will be of interest to determine whether there are differences in the DNA binding activities or mRNA levels of the NF-4FB and AP-1/c-jun gene products in activated versus resting $T$ cells. Because the process of $T$-cell activation has been shown to result in increases in c-fos mRNA levels, and because the $c-f o s$ gene product has recently been shown to associate with, and activate the AP- 1 transcription factor $[19,20]$, it is also possible that de novo c-fos transcription and translation is necessary for $4 \mathrm{~F} 2 \mathrm{HC}$ enhancer activation. This hypothesis is in accord with our previous finding of a requirement for protein synthesis in $4 \mathrm{~F} 2 \mathrm{HC}$ 
gene activation in $T$ cells [12]. Finally the $c-m y c$ and $c-m y b$ proto-oncogenes have recently been reported to be regulated by transcriptional attenuators in their exon 1intron 1 regions $[21,22]$. Given the results of this study, it will be of interest to determine whether these genes also contain intragenic enhancer elements.

The identity of the NF-4FB gene awaits DNA sequence analysis of the isolated cDNA clone. It will be of interest to determine whether this protein is related to other previously described enhancer binding proteins, in particular whether it contains zinc-finger or homeobox DNA binding domains. Because the NF-4FB binding site does not resemble previously described enhancer elements, this gene may define a novel family of transcriptional activators which are involved in up-regulating the expression of other genes which, like the $4 \mathrm{~F} 2 \mathrm{HC}$, are involved in cell activation. It will also be of interest to determine whether NF-4FB mRNA levels can be correlated with 4F2HC enhancer activity which we have shown varies in different tissue culture cell lines.

\section{References}

1. Kelly, K., B. H. Cochran, C. D. Stiles, and P. Leder. 1983. Cell specific regulation of the c-myc gene by lymphocyte mitogens and platelet derived growth factor. Cell 35: 603-610

2. Reed, J. C., J. D. Alpers, P. C. Nowell, and R. G. Hoover. 1986. Sequential expression of protooncogenes during lectin-stimulated mitogensis of normal human lymphocytes. Proc. Natl. Acad. Sci. USA 83: 3982-3986

3. Robb, R. J., A. Munck, and K. A. Smith. 1981. T-cell growth factors: Quantification, specificity and biological relevance. F. Exp. Med. 154: 1455-1474

4. Cotner, T., J. M. Williams, L. Christenson, H. M. Shapiro, T. B. Strom, and J. Strominger. 1983. Simultaneous flow cytometric analysis of human $\mathrm{T}$ cell activation antigen expression and DNA content. F. Exp. Med. 157: 461-472

5. Davis, M. M. and P. J. Bjorkman. 1988. T-cell antigen receptor genes and T-cell recognition. Nature 334: 395-402

6. Alcover, A., D. Ramarli, N. E. Rochardson, H.-C. Chang, and E. L. Reinherz. 1987. Functional and molecular aspects of human $\mathrm{T}$ lymphocyte activation via $\mathrm{T} 3-\mathrm{T} i$ and $\mathrm{T} 11$ pathways. Immunol. Rev. 95: 5-36

7. Haynes, B. F., M. E. Hemler, D. L. Mann, G. S. Eisenbarth, J. Shelhamer, H. S. Mostowski, C. A. Thomas, J. L. Strominger, and A. S. Fauci. 1981. Characterization of a monoclonal antibody (4F2) that binds to human monocytes and to a subset of activated lymphocytes. F. Immunol. 126: 1409-1414

8. Cotner, R., J. M. Williams, L. T. Strom, and J. L. Strominger. 1983. The relationship between early $\mathrm{T}$ cell activation antigens and $\mathrm{T}$ cell proliferation. In Immunopharmacology: 2nd International Symposium. J. E. Hadden, ed. Pergamon Press, Elmsford, NY.pp. 63-68

9. Quackenbuch, E., M. Clabby, K. M. Gottesdiener, J. Barbosa, N. H. Jones, J. L. Strominger, S. Speck, and J. M. Leiden. 1987. Molecular cloning of complementary DNAs encoding the heavy chain of the human $4 \mathrm{~F} 2$ cell-surface antigen: A type II membrane glycoprotein involved in normal and neoplastic cell growth. Proc. Natl. Acad. Sci. USA 84: $6526-6530$

10. Gottesdiener, K. M., B. A. Karpinski, T. Lindsten, J. L. Strominger, N. H. Jones, C. B. Thompson, and J. M. Leiden. 1988. Isolation and structural characterization of the human 4F2 heavy-chain gene, an inducible gene involved in T-lymphocyte activation. Mol. Cell. Biol. 8: 3809-3819

11. Dynan, W. S. and R. Tjian. 1983. The promoter-specific transcription factor Spl binds to upstream sequences in the SV40 early promoter. Cell 35: 79-87

12. Lindsten, T., C. H. June, C. B. Thompson, and J. M. Leiden. 1988. Regulation of 4F2 heavy-chain gene expression during normal human $\mathrm{T}$-cell activation can be mediated by multiple distinct molecular mechanisms. Mol. Cell. Biol. 8: 3820-3826 
13. Singh, H., J. H. LeBowitz, A. S. Baldwin, and P. A. Sharp. 1988. Molecular cloning of an enhancer binding protein: Isolation by screening of an expression library with a recognition site DNA. Cell 52: 415-423

14. Gorman, C. M., L. F. Moffat, and B. H. Howard. 1982. Recombinant genomes which express chloramphenicol acetyltransferase in mammalian cells. Mol. Cell. Biol. 2: 1044-1051.

15. Anger, P., M. Imagawa, R. Chiu, B. Stein, R. J. Imbra, H. J. Rahmsdorf, C. Jonat, P. Herrlich, and M. Karin. 1987. Phorbol ester-inducible genes contain a common cis element recognized by a TPA-modulated trans-acting factor. Cell 49: 729-739

16. Lee, W., P. Mitchell, and R. Tiian. 1987. Purified transcription factor AP-1 interacts with TPA-inducible enhancer elements. Cell 49: 741-752

17. Staudt, L. M., R. G. Clerc, H. Singh, J. H. LeBowitz, P. A. Sharp, and D. Baltimore. 1988. Cloning of a lymphoid-specific $\mathrm{cDNA}$ encoding a protein binding the regulatory octamer DNA motif. Science 241: 577-580

18. Lamph, W. W., P. Wamsley, P. Sassone-Corsi, and I. M. Verma. 1988. Induction of proto-oncogene JUN/AP-1 by serum and TPA. Nature 334: 629-631

19. Chiu, R., W. J. Boyle, J. Meek, T. Smeal, T. Hunter, and M. Karin. 1988. The c-Fos protein interacts with $\mathrm{c}-\mathrm{Jun} / \mathrm{AP}-1$ to stimulate transcription of $\mathrm{AP}-1$ responsive genes. Cell 54: 541-552

20. Sassone-Corsi, P., W. W. Lamph, M. Kamps, and I. M. Verma. 1988. fos-associated cellular p39 is related to nuclear transcription factor AP-1. Cell 54: 553-560

21. Bender, T. P., C. B. Thompson, and W. M. Kuehl. 1987. Differential expression of c-myb mRNA in murine B lymphomas by a block to transcription elongation. Science 237: 1473-1476

22. Bentley, D. and M. Groudine. 1986. A block to elongation is largely responsible for decreased transcription of c-myc in differentiated HL60 cells. Nature 321: 702-706

23. Dignam, J. D., R. M. Lebovitz, and R. G. Roeder. 1983. Accurate transcription initiation by RNA polymerase II in a soluble extract from isolated mammalian nuclei. Nucl. Acids Res. 11: 1475-1489

24. Singh, H., R. Sen, D. Baltimore, and P. A. Sharp. 1986. A nuclear factor that binds to a conserved sequence motif in transcriptional control elements of immunoglobulin genes. Nature 319: 154-158 